\title{
To Enliven Virtual Communities of Practice through Gamification
}

\author{
A. Andrade ${ }^{1, *}$, C.V. Carvalho ${ }^{2}$ \\ ${ }^{1}$ Virtual Campus Lda, Porto, Portugal \\ ${ }^{2}$ GILT, Instituto Superior de Engenharia do Porto, Porto, Portugal
}

\section{Abstract}

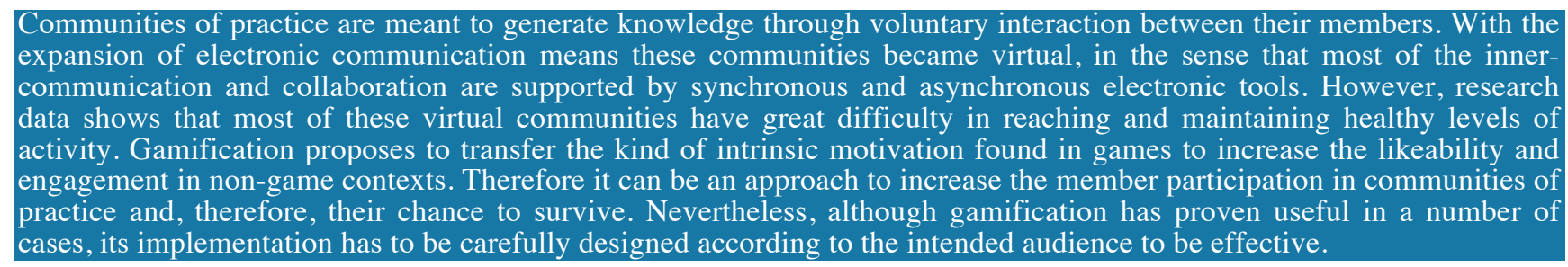

Keywords: Gamification, communities of practice, online communities, motivation, participation.

Received on 30 June 2015, accepted on 30 June 2015, published on 03 July 2015

Copyright (C) 2015 A. Andrade and C.V. Carvalho, licensed to ICST. This is an open access article distributed under the terms of the Creative Commons Attribution licence (http://creativecommons.org/licenses/by/3.0/), which permits unlimited use, distribution and reproduction in any medium so long as the original work is properly cited.

doi: $10.4108 /$ sg.1.4.e6

\section{Introduction}

A Community of Practice (CoP), on Wenger's words, can be defined as a body of individuals with a common set of interests who willingly come together to learn about or to help evolve and mature such interests through collaborative efforts. In other words, it is a group of people, formally or informally organized, who share interest in a particular domain or area and have the specific goal of gaining knowledge related to that field by sharing information and experience within the group [1].

In 1986, Orr documented the fact that co-workers at Xerox organized informal meetings during their lunch time as a form of internal support group, making common problems solving easier for other colleagues. At some point, the company saw the value of such structure and formalized it under the Eureka project [2]. This is the first formal recognition and validation example of a model that would later be denominated as a community of practice, especially since Lave and Wenger published their book on situated learning [3].

One can easily understand the individual interest to participate in such community. But looking at the Xerox example from a business perspective, other organizational benefits come up: through their interaction, employees were actively creating a knowledge repository. This allowed for improved efficiency, reducing any "reinvention of the wheel" which in turn resulted in quicker and better customer service. Having this internal

*Corresponding author. Email: antonio_andrade@virtual-campus.eu knowledge repository also promoted a decrease in the learning curve for organization newcomers. The fact that these experts would frequently get together also promoted a faster resolution of new problems and the spawning of new ideas.

Summing up, a community of practice is not only a platform to retain and access knowledge but also a live medium for the generation of new ideas. This also explains why the idea of $\mathrm{CoP}$ has evolved from an individual learning process to a collective knowledge management approach or organizational learning process, as Brown and Duguid's propose [4], where organizations evolve through informal and/or informal approaches based on extended communities of practice formed by their own employees and, eventually, external contributors to that community.

Wenger identified the three main components for these communities [1]:

- Domain: the knowledge topic common ground that gathers members and community activities. Each member will have a different level of expertise on the domain, spawning from the amateur to the specialist;

- Community refers not only to the isolated members' characteristics but mostly to the fabric of relationships and interaction established between them in the context of the community of practice. A strong sense of belonging will foster interaction.

- Practice represents the shared products and activities in the specific domain, i.e., the core knowledge 
produced by interaction between members. Typical activities engaged inside a community of practice relate to problem solving, information request, experience seeking, asset reuse, coordination and synergy, development discussion and knowledge mapping.

Wenger also describes the evolution of a $\mathrm{CoP}$ as a consequence of the following factors [1]:

- Mutual Engagement: Firstly, through participation in the community, members establish norms and build collaborative relationships; this is termed mutual engagement. These relationships are the ties that bind the members of the community together as a social entity.

- Joint Enterprise: Secondly, through their interactions, they create a shared understanding of what binds them together; this is termed the joint enterprise. The joint enterprise is (re)negotiated by its members and is sometimes referred to as the 'domain' of the community.

- Shared Repertoire: Finally, as part of its practice, the community produces a set of communal resources, which is termed their shared repertoire; this is used in the pursuit of their joint enterprise and can include both literal and symbolic meanings.

Other authors add the following requirements for a healthy community of practice, grouped under three following main topics:

- Social presence: the management of a community of practice often faces many barriers that inhibit individuals from engaging in knowledge exchange. Some of the reasons for these barriers are egos, time constraints and even personal attacks [5]. Thus, communicating with others within a community of practice involves creating social presence [6];

- Motivation: the will to share knowledge is critical to the success of a community of practice [7];

- Collaboration: collaboration is essential to ensure that communities of practice thrive. More seasoned colleagues and a higher educational level tend to foster a more collaborative culture [8].

The evolution of the information technology field and widespread access to internet communications made the online context very interesting for the creation and implementation of virtual communities of practice [9].

Virtual communities of practice complement the traditional co-located communities of practice with the comfort of remote synchronous and asynchronous interaction [10]. Thus members are no longer restricted to being in the same physical space or even time zone to engage in common activities, reducing, for instance, the sense of isolation in professionals who work alone [11]. Furthermore, the new internet tools allow not only for easier organization and access to the shared repository but also enable easier collaboration.

Since virtual communities of practice are very dependent on the technological support layer, they step away from the more spontaneous traditional initiatives and tend to be backed by institutions or organizations making them more of top-down approaches. Although one could see this as a paradox regarding the ease of wide-access, the literature also reports that these virtual communities tend to have a much slower development [7]. In other words, although the online platform development requires the involvement of technical experts and appropriate funding, this technical aspect can be estimated and fine-controlled.

Having people join the community and participating, however, is not so easily controllable. Regular participation throughout the community fabric is a common drawback of virtual communities of practice, especially on their early life [12].

However Song proposes that instead of simply painting "a bleak picture of virtual communities" it is rather necessary to understand that these communities do imply a significant shift in the mode of engagement of individuals [13]. It is therefore important to acknowledge that online communities do not provide the richness of identity hints we find in face-to-face interaction. Personality traits are often emphasized or attenuated, which may also lead to online newcomers' insecurity in participating.

\subsection{Communities of Practice and Learning}

There is a close relation between Communities of Practice and Learning. As stated before, the participation in a CoP is normally motivated by the will to learn more about a certain topic, profiting from the experience and knowledge of the other members. This kind of learning takes normally the form of informal learning and contributes to lifelong learning that is the permanent improvement of our knowledge, skills and competences throughout life.

The impact of lifelong learning in the Knowledge Society has been rendered evident on multiple occasions [14]. Lifelong learning is by definition voluntary and selfmotivated and enhances social inclusion and personal development but also fosters competitiveness and employability. Moreover, lifelong learning initiatives have been part of the European Union policies for more than a decade now [15].

In addition to the individual personal development gains, lifelong learning has also the potential to benefit employing institutions and companies [16]. This has been recognized by human resource managers and training on the job is today a formal part of organizations' lifecycle. However, in all lifelong learning contexts, disengagement has been recognized as one of the main obstacles. In fact disengagement seems to be a transversal issue for learners, employees (at work), customers, etc.

\subsection{Examples of Communities of Practice}

Communities of practice have been applied to diverse environments, including companies and organizations, education, associations and the social sector, as well as the governmental institutions or for international development [17]. To illustrate the diverse applicability of this concept, we will share the example of three communities we have developed in the context of European Lifelong Learning projects:

- The first is TIED Shoe project that aimed to create a virtual training centre for the development of the footwear industry [18]. This platform was built in 
To Enliven Virtual Communities of Practice through Gamification

two layers: the first welcomed every interested participant, with open interaction (in the form of blogs as well as groups and forums), while the second layer supported e-learning courses. The transition between both layers was made seamless, allowing discussion to spawn from one to the other.

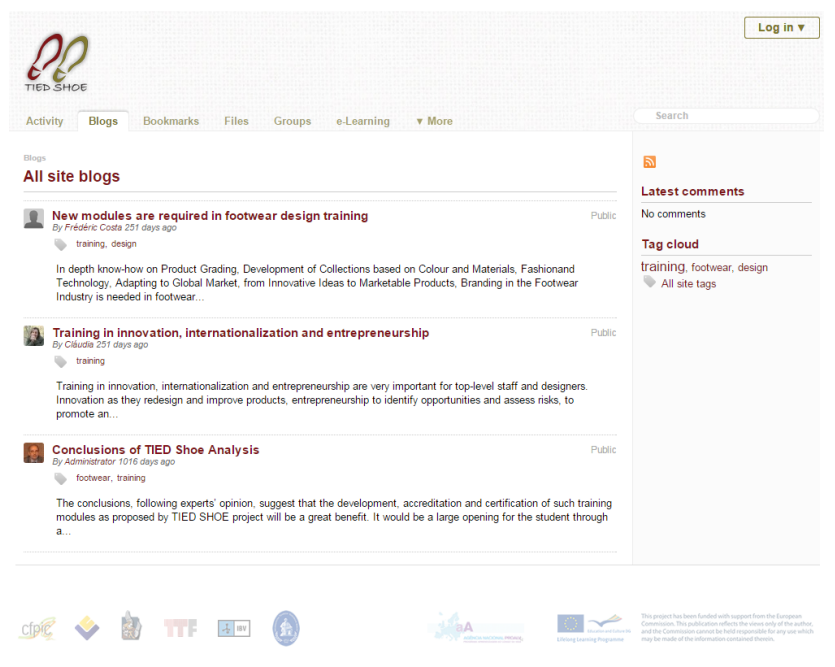

Fig. 1 The TIED Shoe community

- Another project is the European Marketing and Innovation Centres (EMIC) which targeted entrepreneurs and marketers (but also students or innovation professionals) and its general idea was to create a network of national MIC (Marketing \& Innovation Centres). Its main goals were to create an observatory for national good practices (as well as implementing the best results in companies) and support students research and translation to the professional world [19]. Similarly to the TIED Shoe case, two seamless layers were implemented: one for the main social portal and another for the private elearning courses. However, the student support objectives also required the creation of interfaces to relate enterprises and students looking for jobs or internships. MICs were also provided with tools enabling them to design and carry out online surveys.

- Finally we would like to highlight the Serious Game Network. Games are believed to be a potential learning medium [20]. However, looking at the current impact that can be observed from the use of games and simulations as informal medium or optional course support material, serious games have not been explored to their full potential. The SEGAN Network aimed to bring together academic researchers, game producers and Serious Games users but it was also open to any individual interested in the domain, hoping that from their reunion would sparkle more recognition for this learning medium [21].

We have also previously seen the Xerox example and, in 2000 , Verna Allee would suggest that $50 \%$ to $70 \%$ of companies were implementing some kind of knowledge management initiative [22]. This clearly demonstrates the opportunities for the implementation of communities of practice. In [23] several other examples of CoPs are presented, from Research Activities, incubating Creative SMEs, Public Administration, Cancer Surgery, etc.

This article presents an overview of the implementation of communities of practice as lifelong, social-driven, learning support tools. It also discusses the possibility of using gamification for the empowerment of these platforms and finally, presents the results obtained in one of such communities, the Serious Games network CoP SEGAN.

\section{Gamification}

Confirming the issue of the time it takes for a virtual community of practice to reach a natural continuous pace, we have also identified in our experience the lack of engagement or motivation to participate as a major drawback. In particular, this could be seen very clearly on the SEGAN platform. Given the level of attention the network got on its live conferences or even on the Facebook group, we assumed the issue was not the content but perhaps form.

Of course, one can understand the online context is very distracting - that is the nature of the hypertext. Moreover, according to a 2009 research, $43 \%$ of the European internet users were considered as "nonparticipative" [24]. Nevertheless, if the Facebook group got active participation, why couldn't we get some of that engagement in the self-hosted network community? At this point we started looking at what could motivate participation in online communities.

Trevor Moore identified some motivational categories that could explain the participation in virtual communities [25]. The user could simply be altruist, or be looking for a sense of belonging, wisdom, knowledge, or self-esteem, trying to find ways to collaborate, building a reputation or looking for some place to brag and get recognition. This theorization was valuable but did not help us design a plan to engage users in communities.

Eventually we started looking into the concept of gamification and understood that the disengagement issue we were facing had been tackled before in a quite original manner. The motivational category Moore was perhaps missing was the idea of fun which we can find plentifully in the games medium [26]. Games achieve to create what Csíkszentmihályi would, quite famously, designate as flow: a mental state of completely focused motivation [22].

Games, when well designed, create intrinsic motivation. Werbach argues that this kind of motivation can be generated by stimulating three main sensations in the player [23]:

- a sense of autonomy: the player feels in charge as he can choose his challenges, goals and even perhaps his opponents;

- a sense of competence: the player feels he is evolving and is able to achieve new goals, he also is given enough data to measure his success;

- a sense of relatedness: the player is able to compare himself and compete with other individuals and friends; collaboration mechanics can also stimulate motivation.

Gamification brings together marketing strategies and game design thinking to impact on the user experience domain by giving user interfaces a game feeling. Thus, a 
common gamification definitions explains it as the process of applying game mechanics to an interface as means to engage users [29], augmenting the interface's likeability and perhaps virality. Or, as Huotari and Hamari put it, it is "the use of game thinking and game mechanics in non-game contexts to engage users in solving problems and increase users' contributions" [30].

To "gamify" an interface takes a lot of thought. One has to start by analyzing the interface audience and to schematize the actions and behaviours intended to be fostered. Only after having designed the activity cycles should rewards and, more importantly, feedback be implemented. Different frameworks have been developed for the design of a gamified system. [23], [25] and [26] all suggest a sequence of questions the designer should follow to get directions. Chou proposes another framework based on what he calls the Octalysis, a chart of eight axes where core drives are related to game mechanics [27].

Despite some criticism and scepticism, company success stories abound [30] [31], including SAP AG, IBM, Deloitte or even Microsoft [32]. Virtual communities of practice such as StackExchange.com seem to thrive having gamification as an important part of its identity (and not only as an overlay).

Although there is no magic recipe one could automatically apply to a given interface, there are a few common elements to keep in mind [23]:

- points provide a clear feedback upon an action and also allow to visualize the progress made; in some situations they also provide a "win state", ie., achievement of a predefined goal. Points could be related with the sense of competence we have previously seen;

- badges can simply symbolize achievement of a set objective but, more interestingly, they should represent all the system possibilities, allowing the user to choose a goal to work to. This also means badges may represent some kind of status/trophy but also an affirmation of a choice made. Badges could then be related with the sense of autonomy we have previously seen;

- leaderboards represent the social aspect of a system, allowing one or one's team to relate with others. Of course, to a certain extent leaderboards and rankings can also be a source of frustration.

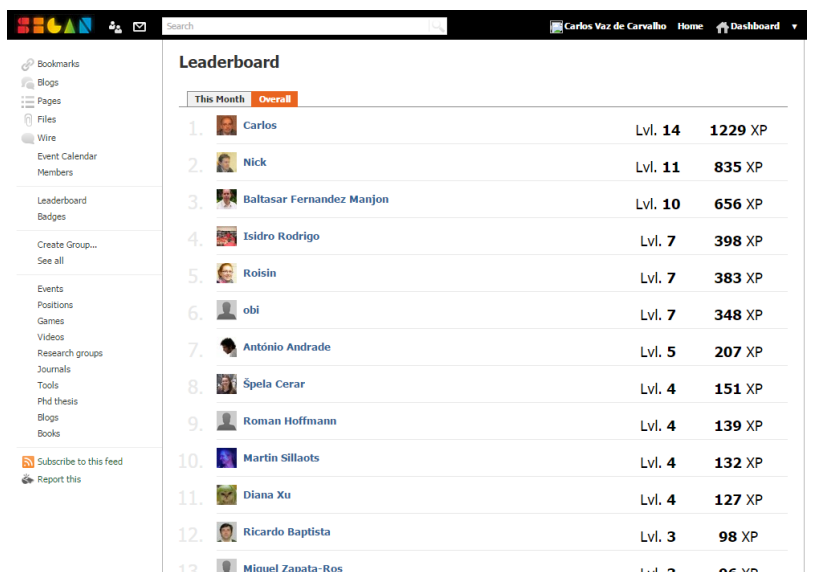

Fig. 2 SEGAN Leaderboard
The most common critique to this process points the risk of turning game-like interaction into an end in itself which creates no implicit motivation, hence undermining content quality and missing out on the experiential and storytelling dimension of a product. In other words, it interprets rewards in a strictly behaviourist way [28]. In fact, helping to make sense of a non-game context, by overcoming this issue, is probably the main objective for gamification [29].

Many of the common gamification techniques seemed to make sense in SEGAN's case and would potentially inject some motivating fun factor. Nonetheless the first step in the process would be to define priorities for the community. Content quality was clearly the main priority to SEGAN but before that it was important to engage members.

Not overwhelming newcomers with gamified metrics was another important factor to take into account. Thus, it was decided that we would use both badges and user experience point (XP) leaderboards. As a general rule, XP tends to value quantity (and long term engagement) while achievement badges value quality. Badges would allow any newcomer to specialize without feeling crushed by the accumulated experience of older members. In practice this means that if visit frequency is a commonly accepted engagement measurement [39], for each day the user logs into the platform he would get 1 XP. However, if a user manages to $\log$ in for thirty consecutive days, she would get the "Enthusiast" badge and the respective XP prize.

Having defined our priorities, contributing contents clearly came out to be the main rewardable action. A blog post is awarded $10 \mathrm{XP}$. Sharing a bookmark would get half that prize. However, if a user happens to create a blog post which receives more than twenty five comments she would be awarded the "Debate Starter" badge. Ideally this duality between achievements and XP allows for multiple ways to feel recognition inside the community.

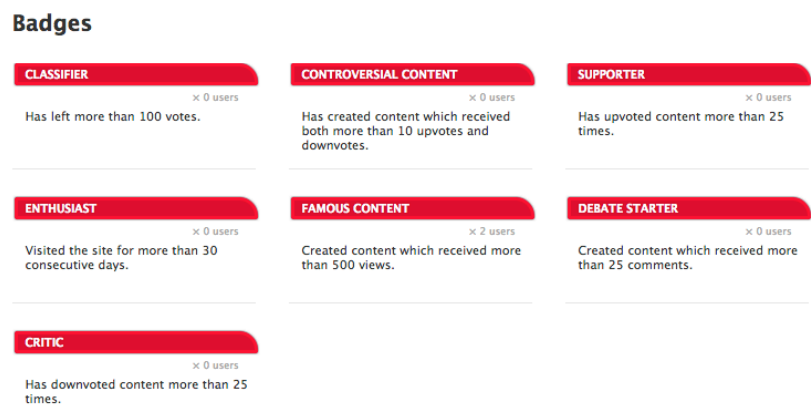

Fig. 3 SEGAN Badges

Content quality is provided by peer review in the simple form of up and down votes. Again, both the voter and the content author are awarded XP and eligible for specific achievement badges.

The data resulting from the community gamification is used throughout the platform to expose interesting users and content. The leaderboard (which stresses monthly gain over all time totals) and badges pages allow to assess overall community performance. Each user's profile is also enhanced with their total XP and badge listing. 


\begin{abstract}
Gamification : Userpoints

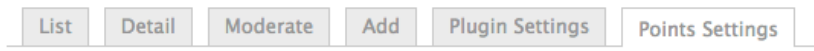

General Activities

Points for making another member a friend:

Points for being made a friend:

Points for each 25 visits to content created:

Points for downvoting content:

Points for upvoting content:

Points for being upvoted on created content:

Points for posting a blog entry:

Fig. 4 SEGAN Gamification administration panel

In addition the users' leaderboards, "Top Rated Content" and "Top Influencers" (users who created most textual content, such as blogs and comments) blocks throughout the platform were also products of this gamification process. Finally, perks for most valuable users and prizes were to be implemented at a future stage.
\end{abstract}

\subsection{Results}

In our approach introducing gamification on the SEGAN platform resulted in a momentarily increase in participation (out of curiosity for the novelty?) but also on criticism. The latter was in fact discomfort for the new presence of leaderboards.

To get a grasp of the community members' perception of these mechanics we setup an online survey (to which we got 16 responses). Most of the users demonstrated positive feelings towards the introduction of gamification elements and the remaining ones were neutral. No one explicitly opposed to gamifying the community. In terms of an engagement improvement, only $20 \%$ of the respondents did not find gamification stimulating.

On a second step we tried to understand which elements users perceived as most stimulating in regards of community participation. The results demonstrated that the first elements we implemented (points and badges) were relevant but also that more dynamic interactions, such as challenges and narratives, would have been appreciated. Finally one should notice the mention of virtual currencies in the responses: perhaps a more direct payback could be a viable option to increase engagement.

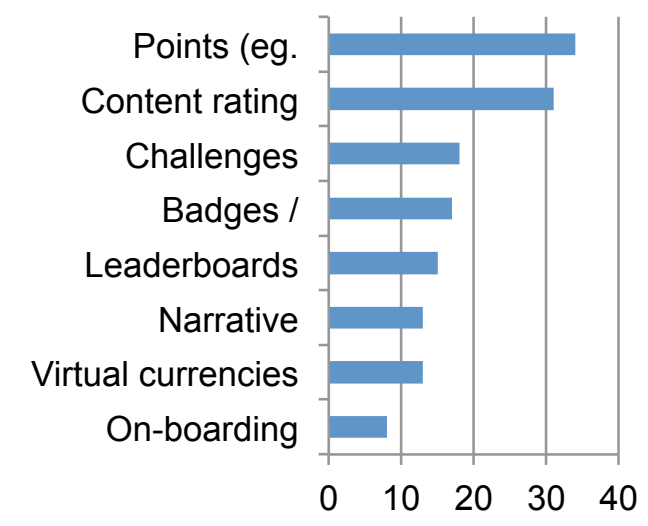

Fig. 5 SEGAN survey: gamification elements ranked by perceived motivation produced.

In addition to the online survey we also conducted interviews to four core members of the community to try and get a better insight over the feel of these new elementsand to get comments on whether gamification could foster participation in VCoPs:

- It can foster because it turns the communities more engaging. Besides, people are social by nature the some gamification elements such as badges and points help people develop a sense of status achievement

- Maybe. But it does not have to be competition (although this is the easiest way to implement game elements). It should be game like activity or game based on collaboration or competition against personal goals.

- A little, but only partially and the effects disappear over time

Other users felt the gamification could have a positive effect on the community but the rules would need some finer balancing. One user in particular took it as mission to demonstrate how points were not at that point correlated with value: he kept repeating actions that required little effort with the intention of gaming the system and easily progress to the top of the leaderboards. This of course would have been much more difficult in a full-fledged community with thousands of members.

Finally, in addition to the specification and design effort, one should also notice that the introduction of game mechanics on an existing platform is often a large overhaul on its technical layer as new commands have to be hooked to most user actions. Hence, that factor should also be considered during the design phase.

\section{Conclusions}

Although there is evidence that communities of practice can be a valuable asset for businesses and general society, depending on the context, its requirement for spontaneous participation can slow down initial developments. And the dependence on voluntary participation of the users also makes these communities (specially the virtual ones) highly volatile. 
As for other media dependent on user-generated content, gamification can be an appropriate and efficient way to foster activity and despite some criticism and failed implementations, successful implementations proved that it can be a significant tool. The main question seems to boil down to the way gamification is implemented in the community.

Our implementation in the SEGAN network resulted in a slightly more active community than the other two, nongamified, VCoPs, despite the scepticism of some of the members and the attempts of gaming the system. But we believe there are some ways the gamification process can be improved to further enliven the community:

- Leaderboards should be made seamless and more useful, i.e., serve as a reputation and identitary distinctiveness;

- Gamification point awards should be more proportional to the community perceived value. This may take us to experiment with a more dynamic award system, at least partially based on a value supply-and-demand economic approach;

- The rewards of the gamified community should also be rendered more tangible through diverse means including the explicit and implicit recognition of the contributions and achievements in exchangeable units.

\section{Acknowledgements}

This work has been partly supported by the European Commission through its European Agency for Culture, Education, Audiovisual in the scope of the Lifelong Learning Programme, KA3 sub-programme, SEGAN network (ref. 519332-LLP-1-2011-1-PT-KA3-KA3NW) and the KA3 project eCity (543573-LLP-1-2013-1-PTKA3-KA3MP).

\section{References}

[1] E. Wenger, Communities of Practice: Learning, Meaning, and Identity, Cambridge University Press, 1998.

[2] J. S. Brown and P. Duguid, "Balancing Act: How to Capture Knowledge Without Killing It.," Harvard Business Review, vol. 78, pp. 73-80, May/June 2000.

[3] M. Wasko and S. Faraj, ""It is what one does": why people participate and help others in electronic communities of practice," Journal of Strategic Information Systems, vol. 9, pp. 155-173, 2000.

[4] C.-H. Tu, "The management of social presence in an online learning environment," International Journal on E-learning, pp. 34-45, April-June 2002.

[5] A. Ardichvili, V. Page and T. Wentling, Motivation and Barriers to Participation In Virtual KnowledgeSharing Communities Of Practice, 2002.

[6] R. Simon and K.-E. Sveiby, "Collaborative climate and effectiveness of knowledge work - an empirical study," Journal of Knowledge Management, vol. 6, pp. 420-433, 2002.

[7] K. W. Lai, K. Pratt, M. Anderson and J. Stigter, Literature Review and Synthesis: Online
Communities of Practice, Dunedin, New Zealand, 2006.

[8] B. Gray, "Informal Learning in an Online Community of Practice," Journal of Distance Education, vol. 19, no. 1, pp. 20-35, Spring 2004.

[9] E. Wenger, N. White and J. D. Smith, Digital Habitats: stewarding technology for communities, 2009.

[10] J. Field, "Is Lifelong Learning Making a Difference? Research-Based Evidence on the Impact of Adult Learning," in Second International Handbook of Lifelong Learning, Part One, Springer Science, 2012, pp. 887-897.

[11] European Union, "Learning for All," 02 Outubro 2012. [Online]. Available: http://ec.europa.eu/education/lifelong-learningpolicy/adult_en.htm. [Accessed Outubro 2012].

[12] I. Chiavenato, Gestão de Pessoas, 2000.

[13] E. Wenger, June 2006. [Online]. Available: http://www .ewenger.com/theory/index.htm.

[14] TIED Shoe, “TIED Shoe Summary," 18 November 2012. [Online]. Available: http://tied-shoe.eu/en/.

[15] EMIC, "EMIC Objectives," 2012. [Online]. Available: http://emic.ismai.pt/objectives/.

[16] C. Vaz de Carvalho and B. Fernandez-Manjon, "Welcome message from the Editors-in-Chief," in EAI Endorsed Transactions on Game-Based Learning, C. Vaz de Carvalho and B. FernandezManjon, Eds., ICST, 2013.

[17] SEGAN, “About SEGAN,” November 2012. [Online]. Available: http://seriousgamesnet.eu/community/pages/view/19 90/about-segan.

[18] V. Allee, "Knowledge Networks and Communities of Practice," OD Practitioner, 2000.

[19] E. Riu and A. Jokisalo, Informal learning in the era of Web 2.0, 2009.

[20] T. Moore, Understanding Member Motivation for Contributing to Different Types of Virtual Communities: A Proposed Framework, 2007.

[21] M. Prensky, "The Motivation of Gameplay, or, the REAL 21st century learning revolution," On The Horizon, vol. 10, no. 1, 2002.

[22] M. Csíkszentmihályi, Flow: The Psychology of Optimal Experience, New York: Harpers Perennial, 1990.

[23] K. Werbach and D. Hunter, For the Win: How Game Thinking Can Revolutionize Your Business, Wharton Digital Press, 2012.

[24] G. Zichermann and C. Cunningham, Gamification by Design, O'Reilly, 2011.

[25] A. Marczewski, “A Simple Gamification Framework / Cheat Sheet," 2012. [Online]. Available: http://marczewski.me.uk/gamificationframework/. [Accessed 4 April 2013].

[26] S. Duffy, "4 Tips for Keeping Your Gamified Community Motivated," April 2012. [Online]. Available: http://mashable.com/2012/04/24/tipsmotivating-gamified-community/.

[27] Y.-k. Chou, "Octalysis: Complete Gamification Framework," 30 April 2013. [Online]. Available: http://www.yukaichou.com/gamification- 
examples/octalysis-complete-gamificationframework/. [Accessed 30 April 2013].

[28] S. Deterding, "Pawned. Gamification and its Discontents," September 2010. [Online]. Available: http://www.slideshare.net/dings/pawnedgamification-and-its-discontents.

[29] S. Nicholson, "Strategies for meaningful gamification: Concepts behind transformative play and participatory museums," in Meaningful Play 2012, Lansing, Michingan, 2012.

[30] G. Zichermann, "7 Winning Examples of Game Mechanics in Action," July 2011. [Online]. Available: http://mashable.com/2011/07/06/7winning-examples-of-game-mechanics-in-action/.

[31] G. Zichermann, "Gamification: The Hard Truths," 23 January 2013. [Online]. Available: http://www.huffingtonpost.com/gabezichermann/gamification_b_2516376.html. [Accessed 2503 2013].

[32] R. E. Silverman, "Latest Game Theory: Mixing Work and Play," 1010 2011. [Online]. Available: http://online.wsj.com/news/articles/SB10001424052 970204294504576615371783795248. [Accessed 01 12 2013].

[33] G. Zichermann, "Getting Three Fs in Gamification," 19 January 2012. [Online]. Available: http://www.gamification.co/2012/01/19/gettingthree-fs-in-gamification/. [Accessed 4 April 2013].

[34] E. Wenger, R. McDermott and W. M. Snyder, Cultivating Communities of Practice, 2002.

[35] D. Shugurensky, The Forms of Informal Learning: Towards a Conceptualization of the Field, 2000.

[36] J. E. Orr, "Narratives at work: story telling as cooperative diagnostic activity," in $C S C W^{\prime} 86$ Proceedings of the 1986 ACM conference on Computer-supported cooperative work, Nova Iorque, 1986.

[37] S. Leino and J. Ovaska, A Survey on Web 2.0, 2008.

[38] T. Kelly, "Everything You'll Need To Know About Gamification," November 2012. [Online].

Available: http://techcrunch.com/2012/11/17/everything-youllever-need-to-know-about-gamification/. 